\title{
THE COLOR OF THE SKIN AS ANALYZED BY SPECTROPHO- TOMETRIC METHODS
}

\section{Apparatus and Procedures}

By CHARLES SHEARD AND LOUIS A. BRUNSTING

(From the Division of Physics and Biophysical Research and the Section on Dermatology and Syphilology, The Mayo Foundation, Rochester)

(Received for publication April 29, 1929)

The color of the skin, to a certain extent, serves as an index of the state of well-being, or the converse, and has been interpreted, after various modes and manners of expression, as evidence of health or disease. On the one hand there is the tanned, full-blooded skin with the texture and sheen of velvet and, on the other hand, the pale, anemic, wrinkled and lifeless skin. To be sure, the normal person exhibits a wide variation or range in the color attributes of the skin, depending on the race, habitude, occupation and exposure to the elements. Without doubt variations in environment or bodily temperature and psychic emotion or unrest play a part in the composite of factors which go to make up the color of the skin. In pathologic conditions, in which there may be abnormalities or derangements of pigmentation and of the vascular distribution (both as to quantity and quality of blood) there is a wide range of variations in the color of the skin. Some of these changes may be recognized readily by the eye; others cannot be so recognized and, if appreciated, cannot be given quantitative estimation by the clinical methods in vogue.

It would seem as though greater accuracy should be introduced in clinical records regarding the color of the skin. This is particularly true in such conditions as jaundice, hemochromatosis, anemia, polycythemia, cyanosis, Addison's disease and in other pigmentary and vascular disturbances in which color plays a part in the diagnosis, and influences judgment regarding favorable or unfavorable progress during the course of treatment.

That there has not been uniformity in correctly or adequately expressing the color of the skin is attested by numerous clinical reports 
in the literature in which are used such expressions as reddish hue, bluish hue, death-like, bronzy, livid, cyanotic, and the like. The language used for expressing conceptions regarding color has been rather figurative or from an artist's viewpoint. This has been due to the lack of standards, and chiefly for the reason that color has not been expressed in terms of its three attributes: relative luminosity (brilliance), dominant wavelength (hue) and purity (saturation).

\section{TINTOMETRIC METHODS}

Various tintometric or colorimetric methods of estimating color have been described in the literature $(2,3,9,13)$. Various color plaques are employed in such tests and are in character analogous to the Tallquist scale for the estimation of hemoglobin. Such tintometric and colorimetric methods of estimating and recording color are doubtless of value to clinical medicine, especially when subjected to some definite system of color analysis, such as the Munsell disk or the Bradley top. However useful such methods may be, they are open to the objection that they do not in any wise analyze the spectral light reflected by the skin and therefore cannot record in terms of the three fundamental attributes of color. Furthermore, pigments and spectral colors are vastly different. The spectral colors of white light, such as sunlight, are pure colors, can be measured as to intensity, purity, and so on, and are, therefore, readily standardized. For instance, spectral yellow of a given wavelength (for example, 590 millimicrons or 5900 Angstrom units) is always the same yellow and can be reproduced exactly.

\section{ATTRIBUTES OF COLOR}

White light can be made spectrally from certain quantities of red, green and blue of limited wavelength values or, again; from certain quantities of orange, green and blue of limited wavelength values according to the tables of Maxwell (7) and others (10). Unless a color is described according to its attributes, nothing definite is stated regarding the color. The three attributes of color are relative luminosity, dominant wavelength and purity. Relative luminosity or brilliance is necessary if color exists at all. It is often spoken of as brightness, tint, value, or visual brightness. In this particular attribute, 
colors may be classified as equivalent to some member of a series of grays of which black and white are the extremes. Relative luminosity simply tells how much of a standard source of light (such as sunlight) the given color is capable of reflecting. By dominant wavelength or hue is meant that attribute of the color which permits it to be classed as reddish, yellowish, greenish or bluish. The hue or tone depends on the wavelength only. Percentage of purity or saturation determines the degree of hue, stating how vivid or distinct it is. Thus the percentage of purity defines how red or how yellow, and so forth, a color is. For a full discussion of these attributes of color and allied topics, reference should be made to the report of the Committee on Colorimetry of the Optical Society of America (14).

Since the pigmentation of the skin is largely due to melanin (at least in brunets, negroes and those suffering from Addison's disease), the degree of pigmentation, which affects the brilliance and purity only, may be thought of roughly as a smoke screen (in some persons very slight and in others very dense) laid down between solar radiation, on the one side, and the blood in the peripheral capillaries on the other side of the dividing medium, the epidermis. In other words, the presence of a pigment, such as melanin, will not affect the dominant wavelength but will influence the relative luminosity and saturation. On the other hand, the presence of abnormally large quantities of blood at the periphery of the body may influence the dominant wavelength and perchance also the other attributes of the color of the skin. The effects of pigmentation and superficial blood are the subjects of the second and third papers of this series dealing with the color of the skin.

It is readily seen, therefore, in a consideration of the color of the skin (which is a semi-opaque body) that systems dependent on color matching with the human eye fail to analyze sufficiently the light reflected from the skin. It is physically impossible for the human eye to estimate color quantitatively and to analyze it according to its attributes. Rowntree and Brown (9) developed a universal skin tintometer, adaptable to clinical medicine, which consists of nine separate color scales. Matchings were made and grades given to various selected areas of skin under conditions least likely to introduce error. These readings were then subjected to the Munsell (8) system of color measurement. 
SPECTROPHOTOMETRIC ANALYSIS OF THE COLOR OF THE SKIN

Spectrophotometry is, without question, the most fundamental method for the analysis of color (4). Through the use of data obtained by spectrophotometric methods, it is possible to describe color in terms of its three attributes, relative luminosity (brilliance), dominant wavelength (hue), and purity (saturation).

The series of investigations reported in this and the following papers is a continuation of the researches instituted by Sheard (11), and Sheard and Brown (12). We shall attempt to present characteristic spectrophotometric curves and tables of data, and their analyses into fundamentals of color as they are affected by changes which occur in

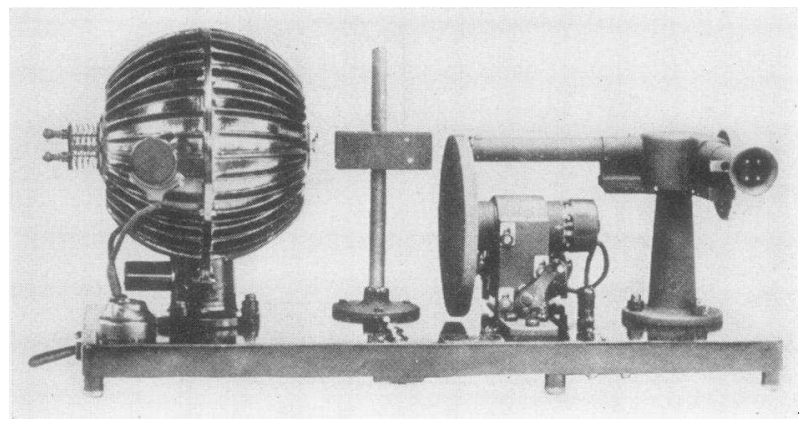

Fig. 1. The Spectrophotometer, or Color Analyzer

disease and as they occur under normal conditions of health, both as regards the part played by pigmentation and that played by superficial blood.

\section{SPECTROPHOTOMETRIC APPARATUS AND METHODS OP PROCEDURE}

The instrument used in these investigations was the direct reading spectrophotometer developed by Keuffel (5) and referred to as the " $\mathrm{K}$ and E color analyzer." Figure 1 is a reproduced photograph of the instrument. Figure 2 is a cross-sectional diagram showing the working parts of the machine and their arrangement as to spectrometric and photometric operations. Quite complete descriptions of the instrument occur elsewhere in the literature (12) but we shall review 
briefly the essential points of construction and operation. A spherical lamp house lined with zinc-oxide paint and holding two 400-watt stereopticon bulbs serves as a source of white light which remains
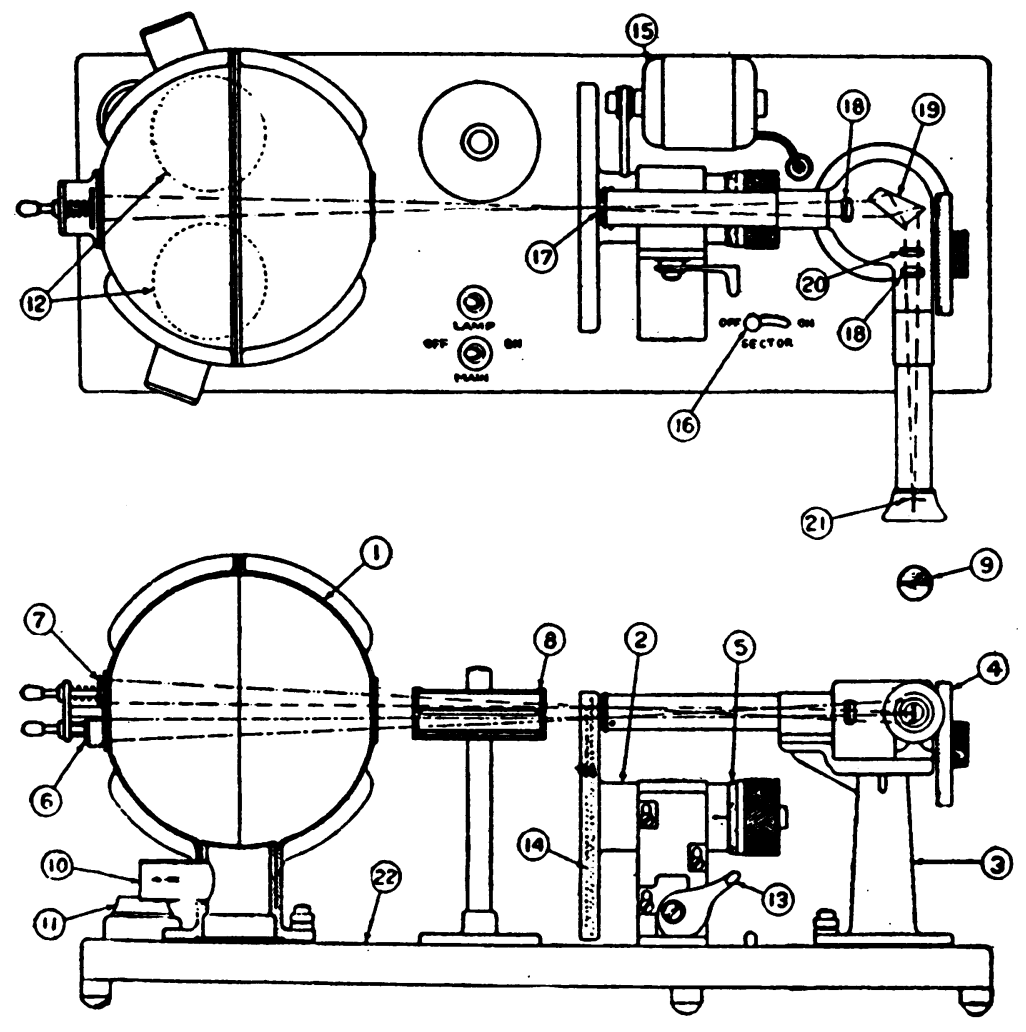

Fig. 2. Cross-sectional Diagrams Showing the Working Parts of the SPECTROPHOTOMETER

1 , spherical light house; 2 , photometer; 3 , spectrometer; 4 , wavelength scale; 5 , photometric scale; 6 , holder for standard sample; 7 , holder for reflection samples; 9 , field of view through the eye slit; 14 , sector disks; 17 , entrance slit; 19 , dispersion prism; and 20 , biprism.

constant during all the readings. Smooth magnesium blocks at the rear of the sphere serve to reflect the light through various transparent mediums (liquids) which may be placed in the path in the support (fig. 2). In such experiments as these, in which an opaque object 
such as the skin is to be studied, the holder for liquids is not used. One of the magnesium blocks is replaced by the area of skin to be investigated. We have recently devised a substitute for the regular attachment to the spectrophotometer which is water-cooled and which enables any portion of the body to be examined. This device will be described in subsequent paragraphs. The two beams of light, reflected from the magesium block and the skin, respectively, enter the spectrophotometer to be analyzed. The spectrometer is essentially the ordinary constant deviation type of instrument except for the addition of a biprism and an observing slit at the exit. The spectrometer is set at any desired wavelength by rotating the wheel, 4, which serves to turn the prism of the spectrometer. The amount of light admitted to match brightness or saturation value is determined by the variation in the sector openings in the rotating disks in the photometer. This is controlled by a calibrated drumhead, 5 . The image at the observing exit slit consists of two semicircular colored fields separated by a horizontal line. The hue of these areas will be the same and will depend on wavelength only. Equality of brightness can be obtained by turning the photometer drumhead until an exact match is obtained. This reading ( 0 to 100 per cent) is then recorded as the percentage of light reflected (or transmitted) at the particular wavelength at which the spectrometer is set. Throughout this particular series of observations the entrance slit was adjusted to give constant reflection of light from the source of white light throughout the whole spectrum, and the entrance and exit slits were thereafter maintained at fixed values.

Readings are taken of the specimen under consideration by turning the calibrated wheel, 4 , over a space of 10 to 20 millimicrons. The percentage of light reflected at each wavelength is read directly from the matching of brilliance obtained by turning the drumhead, 5 , at the same time. A spectrophotometric curve can then be plotted, using wavelengths of light as the abscissas and the percentages of reflection as the ordinates. This gives graphic data which are readily transferable into other values, as will be shown. From such a spectrophotometric curve an experienced observer can determine certain characteristics of the object under consideration by studying the form of the reflection curve in particular regions of the spectrum. Such 


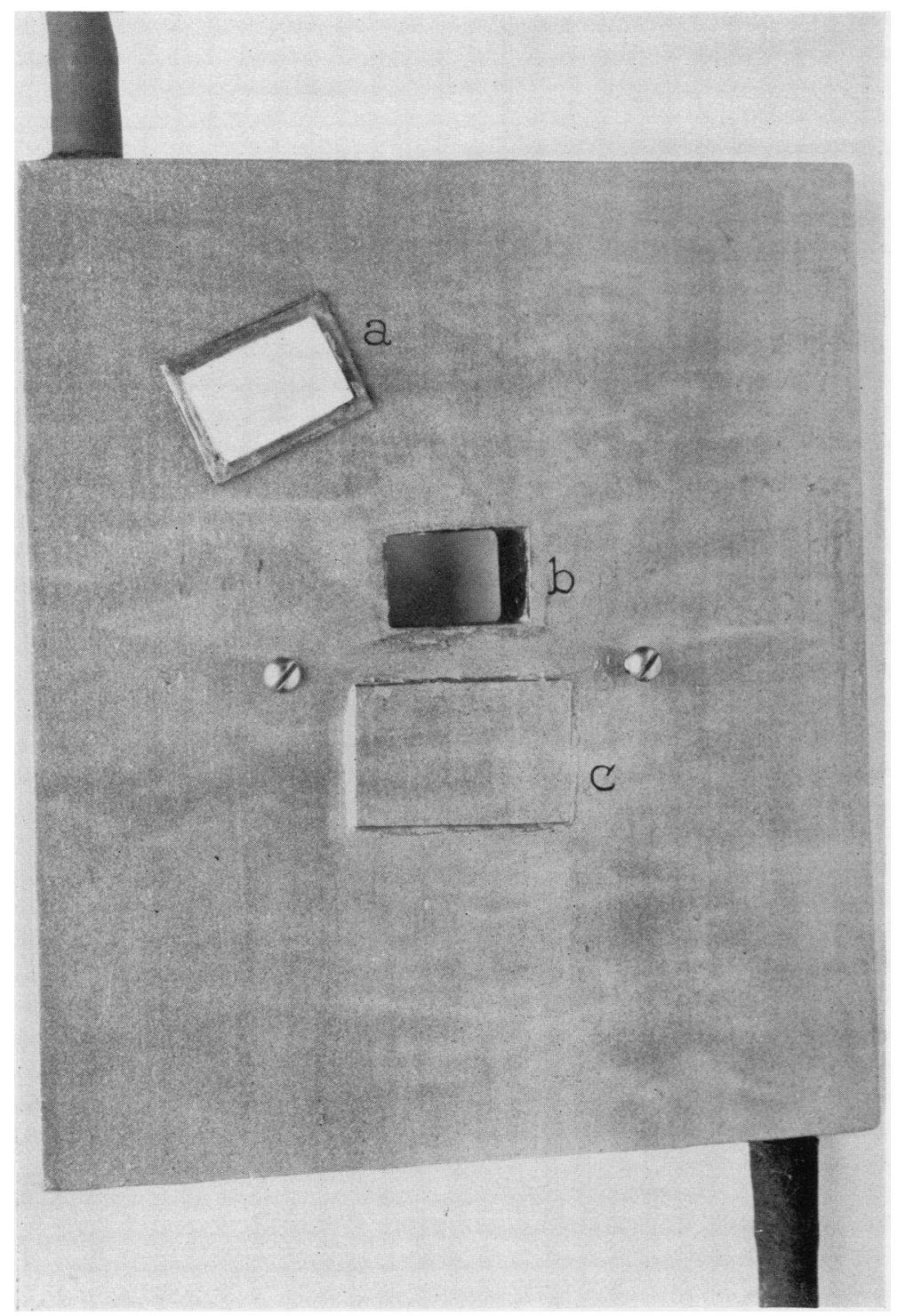

Fig. 3. Water Cell Attachment to Replace Usual Holders for Standard aNd Substance to be Examined by Methods of Reflection

$a$, brass plaque coated with magnesium carbonate; $b$, aperture in the watercooled holder which is attached to the instrument, and $c$, standard in position. 
spectrophotometric readings, since they measure the spectral distribution of the color of the object examined and so actually measure the light stimuli, are independent of the condition of color vision of the observer's eye. Therefore, all observers should get the same results. Also, when a colored object is viewed by the same observer with the naked eye under lights of different quality, the sensation produced under each light will be different although the color itself has not changed physically. Measurements made with a spectrophotometer

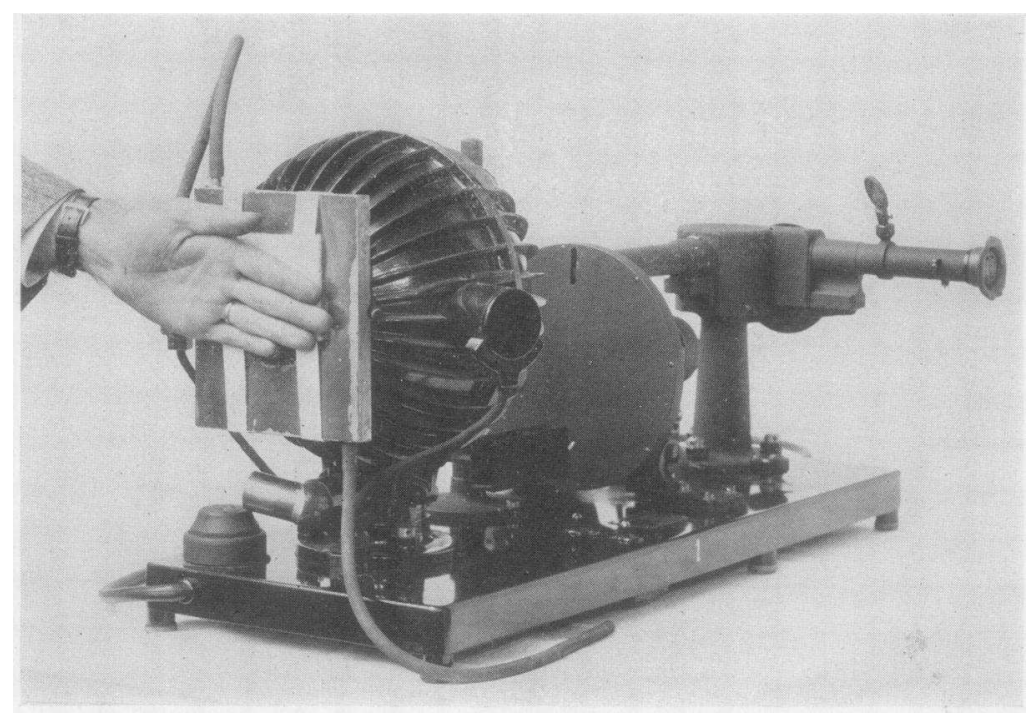

Fig. 4. Keuffel and Esser Color Analyzer with Water Cell Modification Attached; the Relation of the Instrument to Sample to Be Studied Is SHown

are independent of the quality of the source of light as contrasted to color observed by the method of colorimetry or direct matching.

AUTHORS' WATER-COOLED DEVICE TO PERMIT SPECTROPHOTOMETRIC EXAMINATIONS OF LIVING MATERIALS

The Keuffel and Esser color analyzer was originally adapted to record reflection values from such opaque flat objects as paper, cloth, leather, soap, flour, paints, and the like, and to serve as a basis of color 
specification and color control. To adapt the apparatus so that areas of human skin could be measured, it was found necessary to modify the specimen holder. Such easily available portions of the body as the back of the hand or the fingers could be read with the original apparatus, although it was necessary to allow time for the cooling of the lamp-housing in spite of the fact that a current of air was allowed to play between the lamps and the specimen being examined. In devising a practical specimen holder it was borne in mind that although the distance from the source of light could be increased to eliminate to some extent the effects of heat, the illumination suffered in direct proportion. A solution to the problem was found in a thin metal water cell (figs. 3 and 4) so constructed as to fit in the place of the ordinary holders and be directly attached to the spherical lamp-housing. The circulation of the water serves to maintain the cell at a constant cool temperature. Small rectangular perforations are cut into the face of the cell, one above the other. The distal surfaces are covered, in the lower case by a white reflecting surface to take the place of the magnesium block in the original standard. The sample to be tested is brought flush against the upper opening, separated from the metal only by a thin layer of felt which is conveniently changed for purposes of cleanliness and which acts to prevent a change of temperature in the skin. A small, removable plaque, faced on the one side with white magnesium carbonate paint, was constructed and placed in the aperture ordinarily used for reflection purposes in case the instrument was to be used for transmission spectral studies. It is necessary to take the same precaution with the white facings on these metal plaques as with the standard magnesium blocks, for they must be kept smooth and clean throughout. They can be checked at the beginning of any particular experiment to determine whether the reading of brilliance on the sector scale is approximately 100 per cent.

CONVERSION OF SPECTROPHOTOMETRIC DATA INTO TERMS OF COLOR EXCITATION VALUES

In order to convert the curves of spectral reflection (or transmission) into a form which can be interpreted directly in terms of color excitation values, it is necessary to make certain computations. Probably 
the most fundamental of all psychophysical data relating to color are the three color excitation curves which represent graphically the laws of a three-color mixture. The principal data on these relationships we owe to Maxwell (7), Abney (1), and König and Dieterici (6). The results of the latest investigations on this matter, reduced to an equal energy spectrum and referred to average noon sunlight, are plotted in figure 5.

Spectrophotometric data are given generally in the form of curves of spectral transmission or reflection. Such curves require combina-

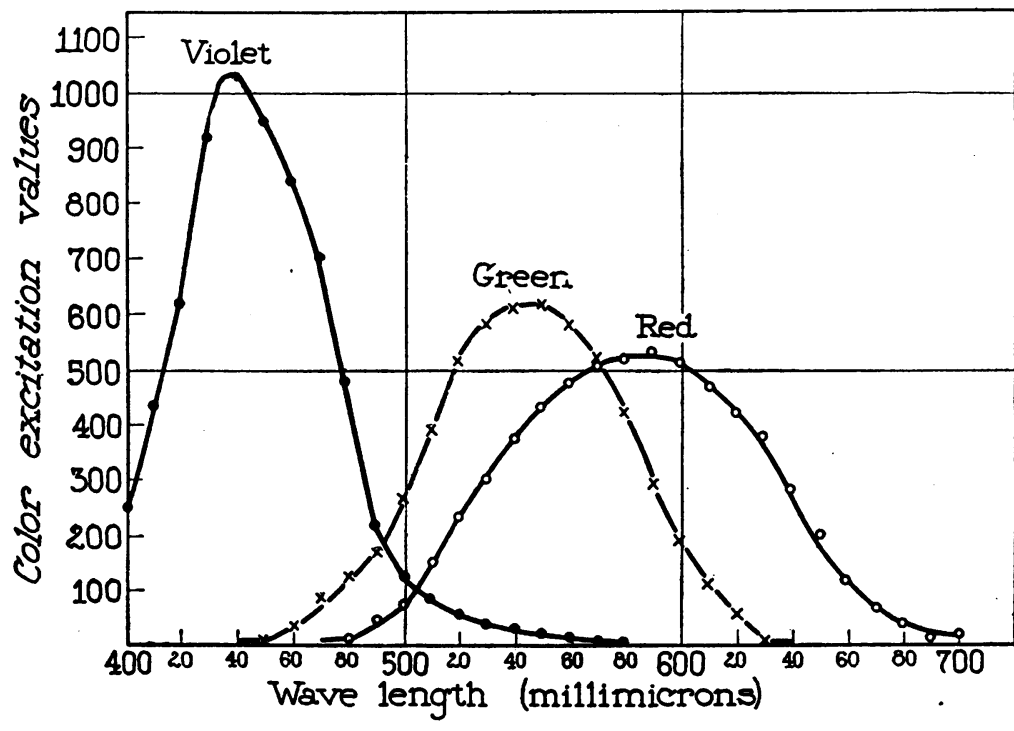

Fig. 5. Elementary Color Excitations for Different Wavelengths

tion with a certain energy distribution representative of the particular source with which the object is illuminated in order that they shall become determinative of a definite color. The process of reducing any given set of spectrophotometric data to excitation color values is therefore as follows: (1) multiply each of the ordinates of the transmission or reflection curve by 'the corresponding ordinate of the energy distribution curve of the source; (2) multiply each of the ordinates of the resulting curve by the corresponding ordinate of each of the color excitation functions as shown in figure 5 , this being a separate opera- 
tion for each of the three excitations and yielding three separate curves which represent the respective excitation values for each wavelength of the given stimulus; (3) determine separately the areas of the three curves thus found, and (4) reduce the three areal values thus obtained to percentage form, so that their determined ratio re-

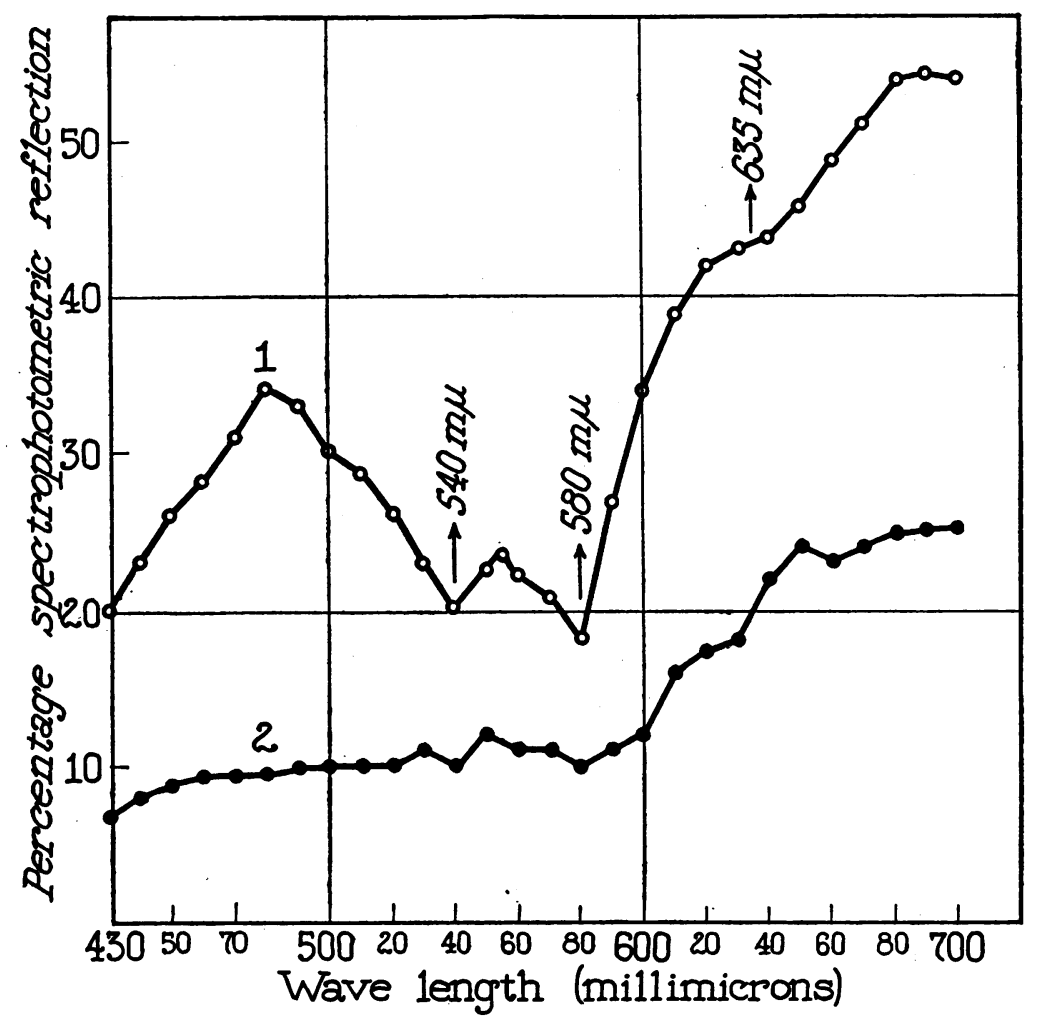

Fig. 6. Spectrophotometric CuRves Obtained by Reflection from the Fingers

Curve 1, normal blond, curve 2, normal mulatto

mains unchanged but their sum becomes equal to 100 . The color excitation values can then be expressed by means of two numbers, representing the red and violet excitation percentages, that for the green being obtainable by subtracting the sum of these two values from 
100. Complete details on these points are given in the report of the Committee on Colorimetry of the Optical Society of America (14).

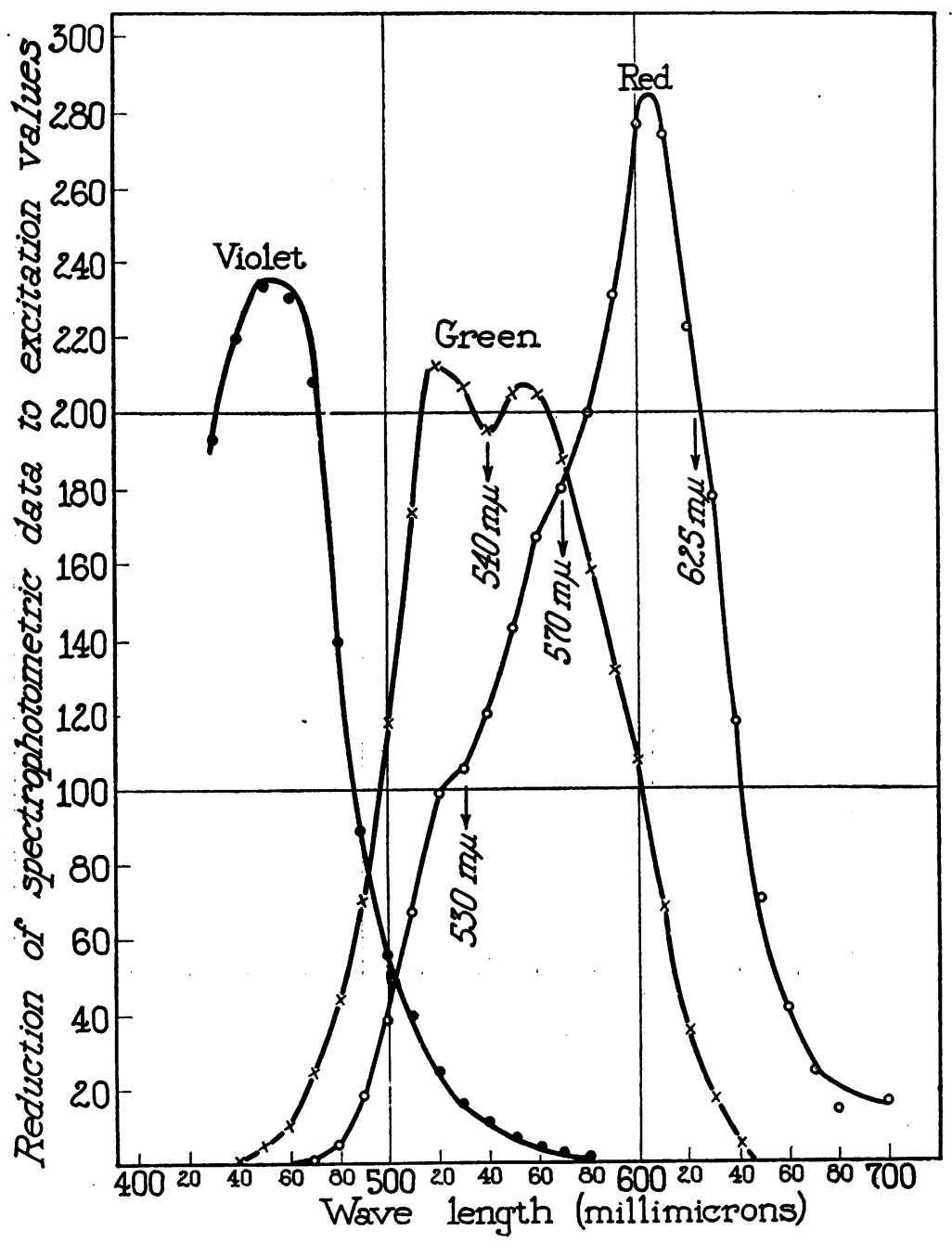

Fig. 7. Analysis of the Spectrophotometric Data (Shown Graphicaliy in Figure 6), into the Three Primary Excitation Curves

Figure 7 contains the red, green and violet excitation curves for the spectrophotometric data given in curve 1 of figure 6 , which was 
obtained in the case of a young normal blond. This curve of spectrophotometric reflection, as well as that which gives the subsequent analysis of this reflection curve into fundamental red, green and violet

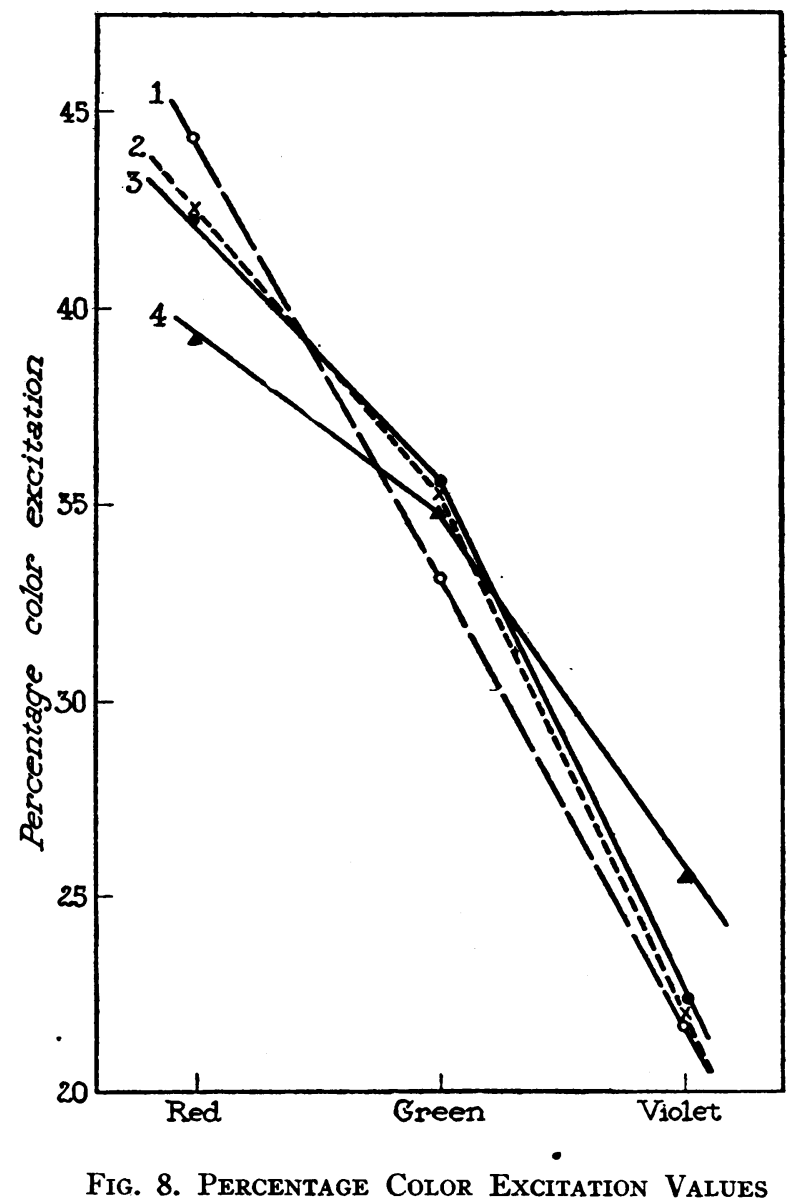

Curve 1, normal brunet; curve 2, a case of Addison's disease; curve 3, a normal negro, and curve 4, a case of scleroderma. All data were obtained from fingers held slightly below heart level.

color values, shows the presence of spectral bands at 625,580 and 540 millimicrons, respectively. The bands at 580 and 540 millimicrons are due to the absorption of light by the blood (oxyhemoglobin bands), 
and the band at 625 millimicrons may be attributed to the presence of hematin in either the skin or the blood, or possibly both. The conversion of the spectrophotometric curves into color excitation

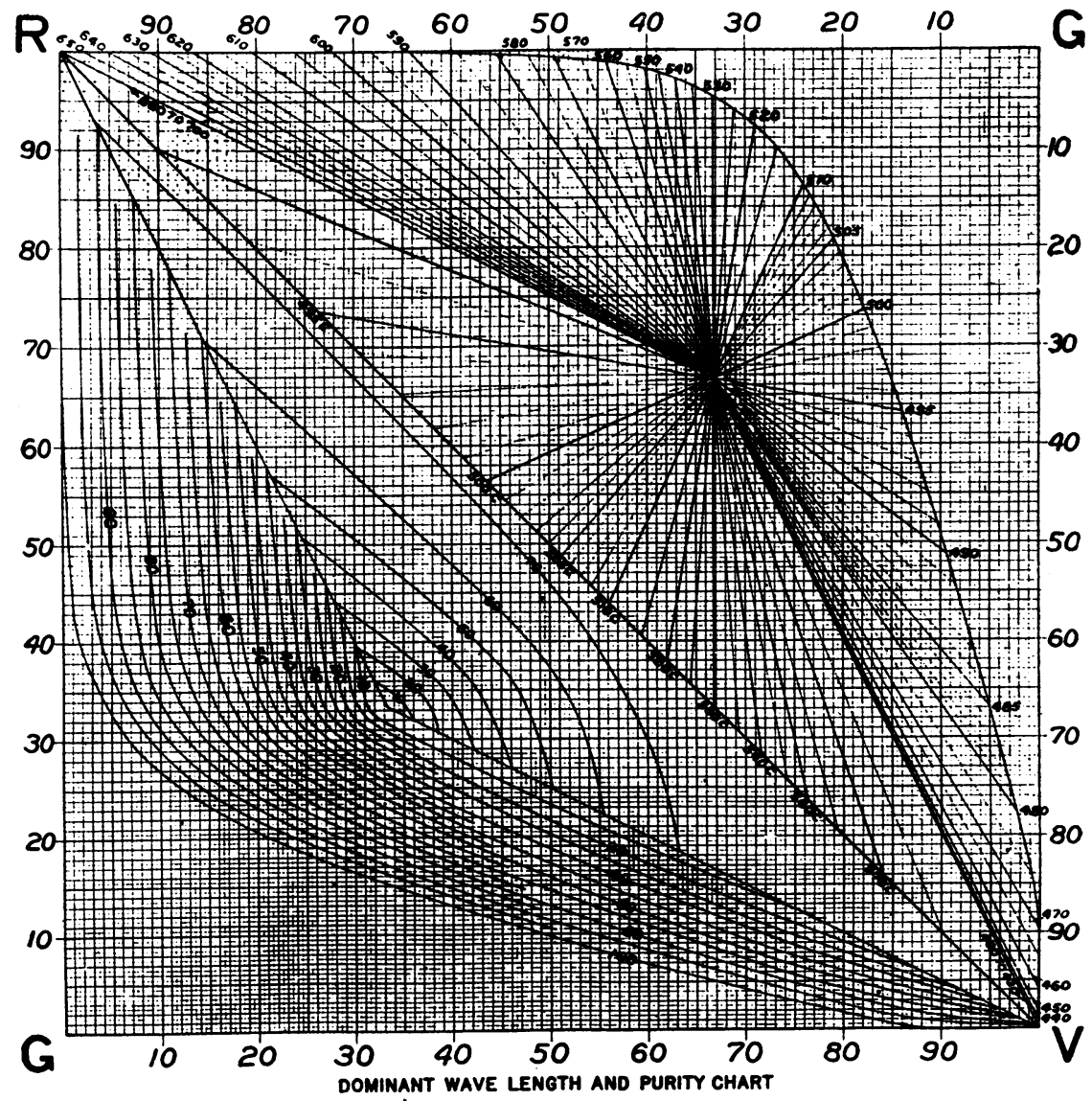

Fig. 9. Dominant Wavelength and Purity Charts from Which the Hue and the Degree of Saturation May Be Obtained by the Use of Data from the Percentage Excitation Calculations

values, as outlined in the preceding paragraph, is much simplified and rendered more rapid by the use of a special color slide rule prepared by Keuffel and Esser (Hoboken, New Jersey) for this purpose.

The summation of the values of the color excitations for the three 
fundamental colors, red; yellow and violet, respectively, proceeding from 700 to 430 millimicrons (for example, the separate excitation color curves shown in figure 7) gives the total red, green and violet excitation values respectively. From the sum of the three separate summation values it is easily possible to obtain the percentage of red, green and violet color excitations, respectively. Figure 8 gives a group of such percentage color excitation values in the case of a normal brunet (curve 1), a case of Addison's disease (curve 2), a normal negro (curve 3) and a case of scleroderma (curve 4). In the subsequent papers which deal with the rôle of pigmentation and of superficial blood a number of such analyses will be presented and discussed.

In addition to the data regarding the total color excitation values and the relative percentages of red, green and violet, respectively, in the white light reflected by the skin, the dominant wavelength or hue and the percentage purity or saturation can be determined by the use of charts provided for this purpose. A reproduction of such a chart. which may be obtained in large dimensions from Keuffel and Esser, is shown in figure 9. Using this color triangle and taking the percentages of red and violet determined from an analysis of any spectrophotometric curve into its fundamental red, green and violet components, we are able to find the dominant wavelengths (or hues) as well as the degrees of purity and relative luminosities.

\section{SUMMARY}

In this paper we have presented and briefly commented on: (1) tintometric methods for estimating the color of the skin; (2) the fundamental attributes of color; (3) spectrophotometric apparatus and methods of procedure for obtaining curves of the reflection, by the skin, of light of various wavelengths; (4) the authors' water-cooled device to enable spectrophotometric determinations to be made on living materials; (5) spectrophotometric analyses of the color of the skin, and (6) conversion of spectrophotometric data into terms of color excitation values, percentages of fundamental red, green and violet reflected by the skin, dominant wavelength, purity and relative luminosity. 


\section{BIBLIOGRAPHY}

1. Abney, W. de W., Researches in Colour Vision and the Trichromatic Theory. London, Longmans, Green and Co., 1913, 431 pp.

2. Freund, Leopold, and Novak, Franz, Wien. klin. Wchnschr., 1925, xxxviii, 760. Neuere Behelfe zur Messung von Hautrötungen und-bräunungen.

3. Freund, Leopold, and Valenta, Eduard, Deutsch. med. Wchnschr., 1922, xlviii, 839. Eine Rötungsskala.

4. Gibson, K. S., Glazebrook's Dictionary of Applied Physics, Macmillan Co., 1923, 737. Spectrophotometry.

5. Keuffel, Carl W., J. Optic. Soc. Am. and Rev. Sci. Instr., 1925, xi, 403. A Direct Reading Spectrophotometer.

6. König, A., and Dieterici, C., Ztschr. f. Psychol. und Physiol. d. Sinnesorg., 1892, iv, 241. Die Grundempfindungen in normalen und anomalen Farbensystemen und ihre Intensitätsverteilung im Spektrum.

7. Maxwell, J. C., Scientific papers, 1, 410. On the Theory of Compound Colors and the Relations of the Colors of the Spectrum.

8. Munsell, A., A Color Notation. Baltimore, Munsell Color Co., 6th Ed., 1923.

9. Rowntree, L. G., and Brown, G. E., Am. J. Med. Sci., 1925, clxx, 341. A Tintometer for the Analysis of the Color of the Skin.

10. Sheard, Charles, Physiological Optics. Chicago, Cleveland Press, 1918, 513 pp.

11. Sheard, Charles, Science, 1926, lxiv, 70. Analysis of the Color of the Skin and Its Significance.

12. Sheard, Charles, and Brown, G. E., Arch. Int. Med., 1926, xxxviii, 816. The Spectrophotometric Analysis of the Color of the Skin and the Observations by This Method in Normal and in Pathologic Subjects.

13. Todd, T. W., and Van Gorder, L., Am. J. Phys. Anthrop., 1921, iv, 239. The Quantitiative Determination of Black Pigmentation in the Skin of the American Negro.

14. Troland, L. T., J. Optic. Soc. Am. and Rev. Sci. Instr., 1922, vi. 527. Report of Committee on Colorimetry. 